\title{
LY294002 enhances cytotoxicity of temozolomide in glioma by down-regulation of the PI3K/Akt pathway
}

\author{
LINGCHAO CHEN ${ }^{1}$, LEI HAN ${ }^{2,3}$, ZHENDONG SHI $^{2,3}$, KAILIANG ZHANG ${ }^{2,3}$, YANWEI LIU ${ }^{4}$, \\ YONGRI ZHENG ${ }^{1}$, TAO JIANG ${ }^{4}$, PEIYU PU ${ }^{2,3}$, CHUANLU JIANG ${ }^{1}$ and CHUNSHENG KANG ${ }^{2,3}$ \\ ${ }^{1}$ Department of Neurosurgery,The Second Affiliated Hospital of Harbin Medical University, Harbin 150086; \\ ${ }^{2}$ Department of Neurosurgery, Tianjin Medical University General Hospital, Laboratory of Neuro-Oncology, \\ Tianjin Neurological Institute, Tianjin 300052; ${ }^{3}$ Key Laboratory of Neurotrauma, Variation and Regeneration, \\ Ministry of Education and Tianjin Municipal Government, Tianjin 300060; ${ }^{4}$ Department of Neurosurgery, \\ Tiantan Hospital, Capital Medical University, Beijing 100050, P.R. China
}

Received August 31, 2011; Accepted November 4, 2011

DOI: $10.3892 / \mathrm{mmr} .2011 .674$

\begin{abstract}
The introduction of temozolomide (TMZ) has advanced chemotherapy for malignant gliomas. However, a considerable number of glioblastoma (GBM) cases are refractory to TMZ. Previous studies have revealed that the PI3K/ Akt pathway is activated in an ataxia telangiectasia and $\operatorname{Rad} 3$ related-dependent manner in response to TMZ. Thus, we hypothesized that PI3K inhibitors may act as antitumor agents against gliomas and potentiate the cytotoxicity of TMZ. The cytotoxicity of a PI3K inhibitor, LY294002, was examined both alone and in combination with TMZ in human glioma cell lines. Proliferation of tumor cells treated with LY294002 in combination with TMZ was significantly suppressed compared to treatment with either drug used alone. The combination treatment induced a higher apoptosis rate, while reducing the invasive capability of U87 cells. The apoptosis-associated proteins, cleaved-caspase- 3 and Bax, were more significantly up-regulated by the combined treatment than by TMZ used alone. In addition, p-Akt and Bcl-2, which can promote TMZ resistance, were markedly decreased by LY294002. These findings suggest that LY294002 enhances the cytotoxicity of TMZ by down-regulation of the PI3K/Akt pathway.
\end{abstract}

Correspondence to: Dr Chuanlu Jiang, Department of Neurosurgery, The Second Affiliated Hospital of Harbin Medical University, 246 Xuefu Road, Nangang, Harbin 150086, P.R. China

E-mail: jc16688@163.com

Dr Chunsheng Kang, Department of Neurosurgery, Tianjin Medical University General Hospital, Laboratory of Neuro-Oncology, Tianjin Neurological Institute, 152 Ahshan Road, Heping, Tianjin 300052, P.R. China

E-mail:kang97061@yahoo.com

Key words: glioma, LY294002, temozolomide, Akt

\section{Introduction}

Glioblastoma (GBM), the highest grade glioma, is the most common malignant primary brain tumor in adults. Despite the development of aggressive treatments, the median survival rate for patients diagnosed with GBM has only marginally changed over the past 25 years and remains approximately one year (1-4). Thus, there is an urgent need to develop novel therapeutic approaches to cure this malignancy. As the molecular mechanisms of gliomas have gradually been clarified, improving chemotherapeutic methods is the key to combination treatment (3,5-11). The therapeutic goal of chemotherapy is to trigger tumor-selective cell death. Compared with necrosis, programmed cell death can be better induced by chemoagents.

Temozolomide (TMZ) is a novel oral chemotherapeutic drug with a broad-spectrum antitumor activity which penetrates the brain and does not require hepatic metabolism for activation $(2,12)$. Roos et al reported that TMZ triggered malignant glioma cell apoptosis by induction of DNA O6-methylguanine (13). However, using TMZ alone would induce drug resistance in tumors and decrease the ratio of efficacy to price $(12,14-16)$. As is well known, p-Akt, EGFR and Bcl-2 apoptosis-associated protein activation is one of the mechanisms of TMZ resistance $(14,17)$. Previous studies have shown that LY294002, which suppresses PI3K, can inhibit the expression of p-Akt in cancer (18-20). Our aim was to ascertain whether LY294002 increases the cytotoxicity of TMZ in vitro.

In the present study, TMZ combined with LY2940042 more significantly inhibited tumor cell growth and invasive ability compared with TMZ used alone. In addition, the combination therapy induced higher rates of apoptosis than single therapy. The mechanism of the two drugs was mediated by the up-regulation of cleaved-caspase- 3 and Bax, decreasing the expression of $\mathrm{p}-\mathrm{Akt}$ and $\mathrm{Bcl}-2$.

\section{Materials and methods}

Tumor cell line. Human GBM cell line U87 was purchased from the Chinese Academy of Sciences Cell Bank. The cells 
were maintained at $37^{\circ} \mathrm{C}$ in a $5 \% \mathrm{CO}_{2}$ incubator in Dulbecco's modified Eagle's medium (DMEM) supplemented with $10 \%$ fetal bovine serum (FBS).

Reagents. TMZ was kindly supplied by Tasly Pharmaceutical Co., Ltd. (Tianjin, China). LY294002 was purchased from Sigma Chemical Co. (St. Louis, MO, USA). TMZ and LY294002 were dissolved in $10 \%$ dimethyl sulfoxide (DMSO; Sigma) to make a stock solution (TMZ: $100 \mathrm{mg} / \mathrm{ml}$; LY294002: $100 \mathrm{mg} / \mathrm{ml})$. DMSO concentration was kept below $0.1 \%$ in all the cell cultures and did not exert any detectable effect on cell growth or cell death. MTT [3-(4,5-dimethylthiazol-2-yl)-2,5-diphenyltetrazolium bromide] was purchased from Sigma. Rabbit anti-human polyclonal antibodies against PI3K, p-Akt, Bcl-2, cleaved-caspase-3 and GAPDH; and the horseradish peroxidase-conjugated secondary antibody (goat anti-rabbit) were purchased from Santa Cruz Biotechnology (Santa Cruz, CA, USA).

Cell viability assay. The cytotoxicity of TMZ and LY294002 on U87 cells was determined by MTT assay. Tumor cells were seeded at $2 \times 10^{3}$ cells/well $(0.1 \mathrm{ml})$ in 96 -well flat bottom plates and incubated overnight at $37.1^{\circ} \mathrm{C}$. After exposure to the above described treatment for $24,48,72$, and $96 \mathrm{~h}$, MTT $(0.5 \mathrm{mg})$ was added to each well every $4 \mathrm{~h}$ before the end of the incubation, when the MTT reaction was stopped by adding $10 \%$ SDS- $0.1 \mathrm{~mol} / \mathrm{l} \mathrm{HCl}$. Formazan crystals in the cells were dissolved in DMSO and then the absorbance at $490 \mathrm{~nm}$ was measured using a microplate reader (Bio-Tek ELX800UV, Bio-Tek Instrument Inc., Winooski, VT, USA). All experiments were repeated in triplicate.

Apoptosis assays. U87 cells were plated in 6-well plates and transfected with Oligonucleotide. The apoptosis ratio was analyzed $48 \mathrm{~h}$ post-transfection using the Annexin V-FITC Apoptosis Detection kit (BD Biosciences, San Diego, CA, USA) according to the manufacturer's instructions. Annexin V-FITC and propidium iodide (PI) double staining was used to evaluate the percentage of apoptosis. Annexin $\mathrm{V}^{-} /$ $\mathrm{PI}^{-}$cells were used as controls. Annexin $\mathrm{V}^{+} / \mathrm{PI}^{-}$cells were designated as apoptotic and Annexin $\mathrm{V}^{+} / \mathrm{PI}^{+}$cells as necrotic. Tests were repeated in triplicate.

In vitro invasion assays. Transwell membranes coated with Matrigel (BD Biosciences, San Jose, CA, USA) were used to assay the invasive ability of glioma cells in vitro. Transfected cells were plated at $5 \times 10^{4}$ per well in the upper chamber in serum-free medium. FBS $20 \%$ was added to the medium in the lower chamber. After incubating for $24 \mathrm{~h}$, non-invading cells were removed from the top well with a cotton swab while the bottom cells were fixed with $3 \%$ paraformaldehyde, stained with $0.1 \%$ crystal violet, and photographed in three independent 10x fields for each well. Three independent experiments were conducted and used to calculate fold migration relative to the blank control while the error was calculated as the standard error (SE).

Western blotting. For Western blotting, protein was abstracted from the treated cells. After the protein concentration was determined, equivalent amounts of proteins were boiled in sample buffers with DL-Dithiothreitol (DTT) and then concentrifuged at $12,000 \mathrm{rpm}$ for $15 \mathrm{~min}$ at $4^{\circ} \mathrm{C}$. The protein was separated by SDS-PAGE (PAGE: 15\% gel for MAP1LC3; $10 \%$ gel for Beclin1). Separated proteins in gel were transferred onto PVDF membranes by an electroblot apparatus (Bio-Rad, Hercules, CA, USA). Filters were blocked for $2 \mathrm{~h}$ in 5\% low-fat milk and incubated with rabbit anti-human antibody for PI3K/Akt/p-Akt/Bcl-2/cleaved-caspase-3/Bax and GAPDH at $4^{\circ} \mathrm{C}$ for $12 \mathrm{~h}$. Membranes were then washed with PBS-T and incubated with HRP-conjugated anti-goat antibody for $1 \mathrm{~h}$. Specific signals were detected from the quantitative gel and Western blot imaging system (Becton Dickinson, Franklin Lakes, NJ, USA).

Statistical analysis. Statistical analyses were performed using SPSS version 13.0 software (SPSS Inc., Chicago, IL, USA). Multiple groups were compared using analysis of variance (ANOVA) followed by post Fisher's least significant difference (LSD) testing where appropriate. Data were considered statistically significant at $\mathrm{P}<0.05$. All data are expressed as the mean \pm standard deviation $(\mathrm{SD})$.

\section{Results}

Reduction in the survival of human glioma cells after treatment with LY294002. To clarify the activity of LY294002 as a single antitumor agent, we first performed an MTT assay using U87 cells treated with LY294002 at concentrations of 5-20 $\mu \mathrm{M}$. We found that LY294002 induced a dose-dependent reduction in the proliferation of these cells (Fig. 1A).

We then investigated whether the invasive capability of LY294002-treated glioma cells decreased. The transwell analyses demonstrated that the number of invasive cells were significantly reduced compared with the control group (Fig. 1B). The anti-apoptosis p-Akt has been reported to be associated with PI3K, and we confirmed that LY294002 decreased the levels of PI3K and phosphorylated Akt in cells treated with LY294002 (Fig. 1C). We then examined whether LY294002 induces apoptosis in U87 cells using flow cytometry and double staining with Annexin V (an apoptosis marker) and PI (a DNA-staining dye). The population of apoptotic cells was larger among the U87 cells treated with LY294002 than among the untreated cells (Fig. 1D).

Sensitization of human glioma cells to TMZ. As shown in Fig. 2, the proliferation of cells treated with TMZ was suppressed with LY294002. This LY294002-induced potentiation of the effects of TMZ was recognized at a lower concentration than that needed to induce cytotoxicity with TMZ alone. Isobologram analyses indicated that the effects of the combination of LY294002 and TMZ in both U87 and U251 cells were synergistic. In addition, MTT assay showed that the combination treatment with TMZ and LY294002 clearly reduced the proliferation of both U87 and U251 cells compared with proliferation in the single therapy groups.

Treatment with the combination of TMZ and LY204002 suppresses the cell invasive capability of glioma cells. Results of the cell invasion analysis as shown in Fig. 3 indicate that after different treatments for $48 \mathrm{~h}$, inhibition of cell invasion 
A

C
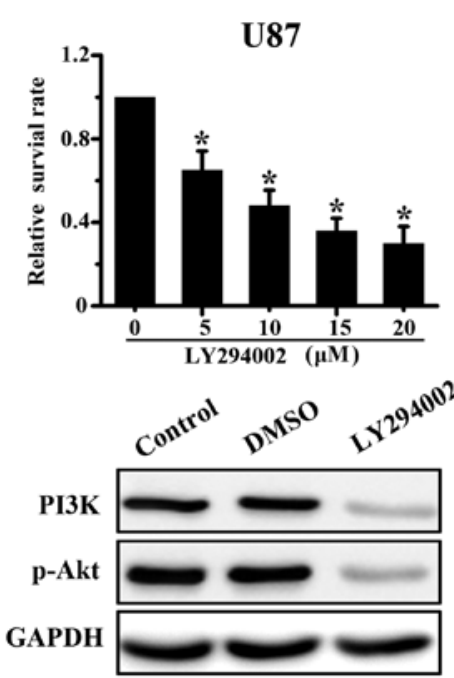

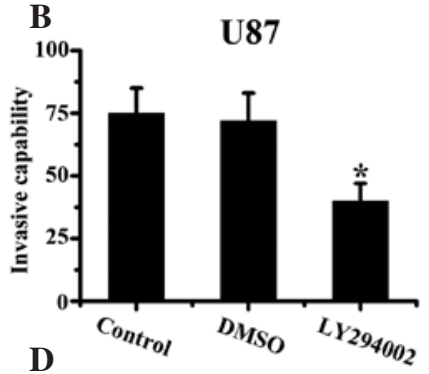

U87

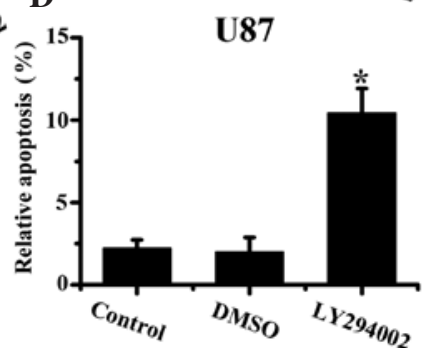

Figure 1. LY294002 inhibits the survival of human glioma in vitro. (A) Relative proliferation of U87 glioma cells treated with different doses of LY294002 as determined by MTT. (B) Transwell assay showed that the invasive capability of U87 cells treated with LY294002 (10 $\mu \mathrm{M})$ was weaker than that with DMSO and the control. (C) U87 glioma cells were treated with LY294002 $(10 \mu \mathrm{M})$ and DMSO, respectively. PI3K, p-Akt and GAPDH expression was determined $48 \mathrm{~h}$ following treatment as assessed by Western blot analysis. (D) Annexin V analysis showed that U87 glioma cells treated with LY294002 displayed greater apoptosis than the other two groups. Data are from one of three representative experiments. " $\mathrm{P}<0.05$ compared with control group.

A

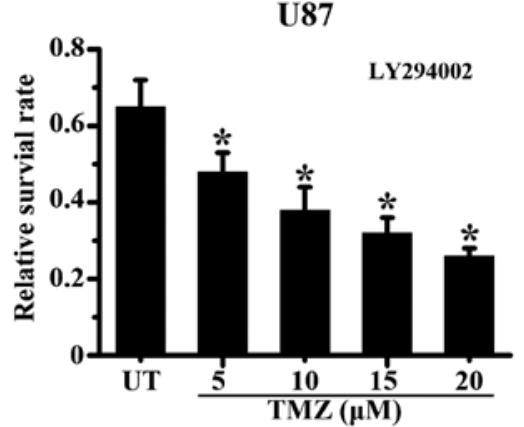

B

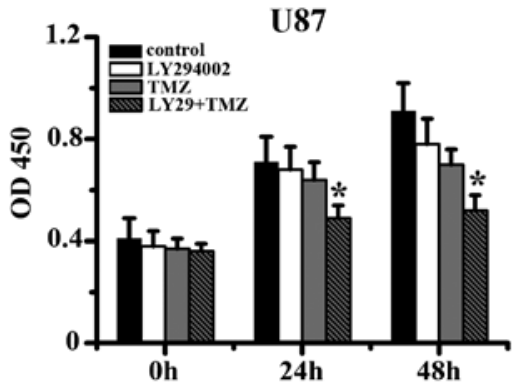

U251

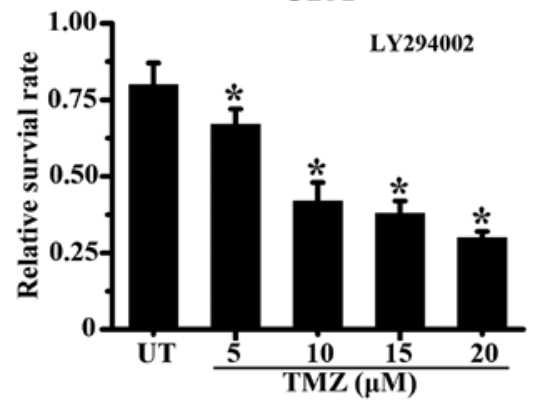

U251

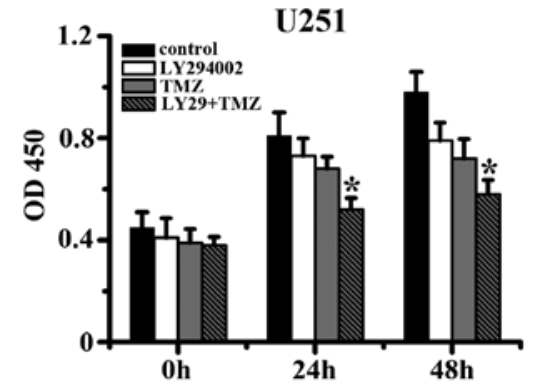

Figure 2. LY294002 increases TMZ-induced proliferation in glioma cells. (A) Relative proliferation of U87 and U251 cells treated with LY294002 and different dose of TMZ as determined by MTT. (B) MTT assay showed the proliferation of U87 and U251 cells treated with LY294002, TMZ and the combination therapy. ${ }^{*} \mathrm{P}<0.05$ compared with control group.

A

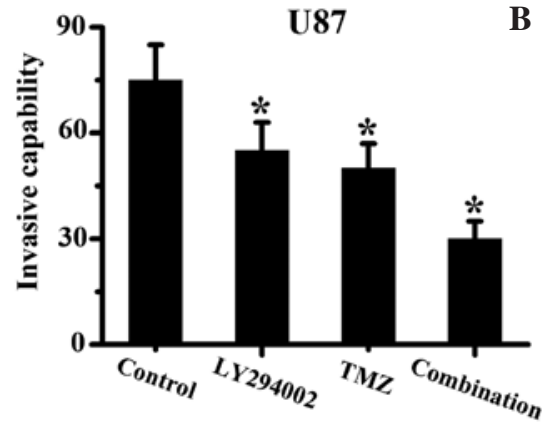

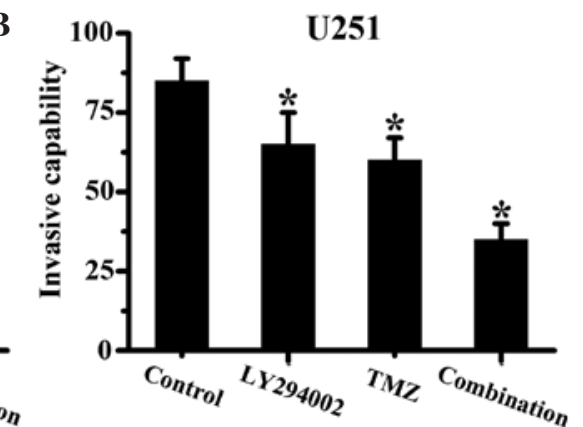

Figure 3. LY294002 increases TMZ-induced invasion in glioma cells. Transwell assay showed the invasive capability of (A) U87 and (B) U251 cells treated with LY294002, TMZ and the combination therapy. ${ }^{*} \mathrm{P}<0.05$ compared with control group. 
A

B
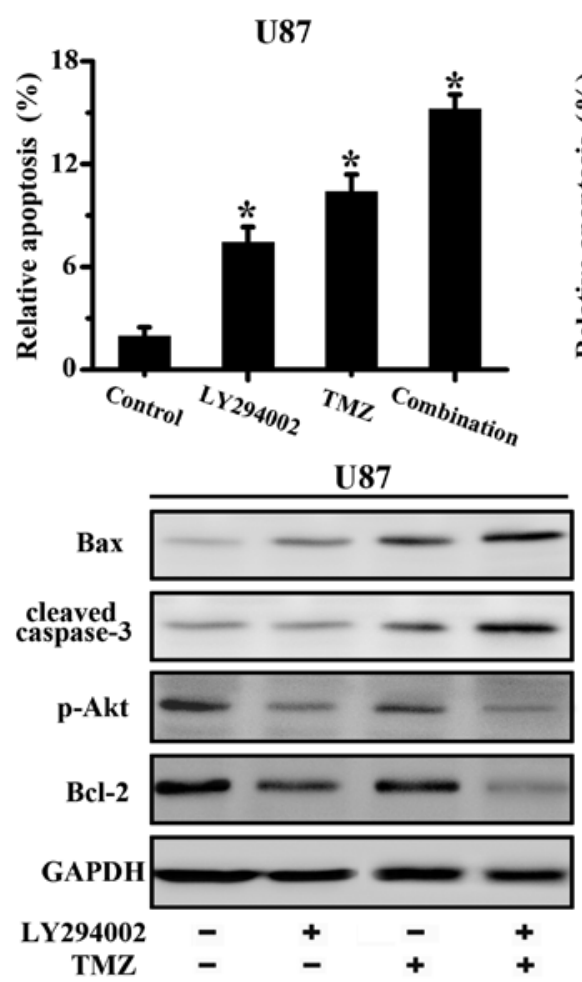

U251

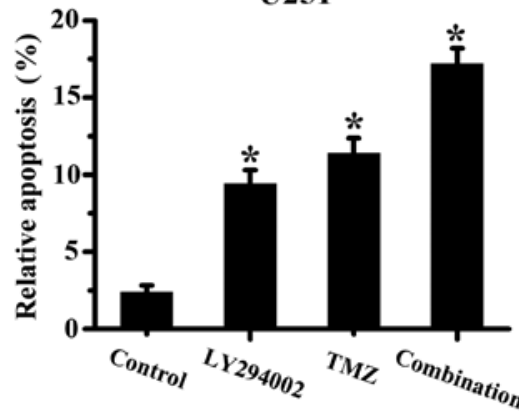

U251

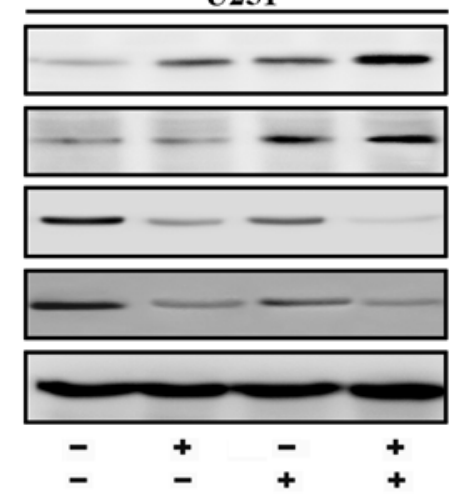

Figure 4. LY294002 increases TMZ-induced apoptosis in glioma cells (A) Annexin V analysis showed that U87 and U251 glioma cells treated with the combination of LY294002 and TMZ displayed greater apoptosis than the other three groups. (B) Bax, cleaved-caspase-3, Bcl-2, p-AKT and GAPDH expression were determined $48 \mathrm{~h}$ following Western blot analysis. Data are from one of three representative experiments. " $\mathrm{P}<0.05$ compared with control group.

in the TMZ and LY294002 group was more notable than that in the TMZ or LY294002 single therapy groups; furthermore, the number of invasive cells in the single therapy group were less than those of the control group.

Treatment with the combination of TMZ and LY294002 induces the cell apoptosis of glioma cells. For Annexin V and PI staining detection with flow cytometry, the percentage of cells in the Q4 quadrant represents the early-apoptosis rate. As shown in Fig. 4A, after 3 days of treatment with the combination of TMZ and LY294002 a significant increase in the number of apoptotic cells was noted compared to that when LY294002 and TMZ were used alone. The results indicate that LY294002 enhances TMZ-induced apoptosis.

Treatment with the combination of TMZ and LY294002 increases the expression of cleaved-caspase-3 and Bax, and decreases $p$-Akt and Bcl-2. Cleaved-caspase-3 and Bax are two important apoptosis-associated proteins in the intrinsic apoptosis pathway. We detected cleaved-caspase-3 and Bax protein expression after the different treatments as assessed by Western blotting. The results are shown in Fig. 4B. Following 3 days of treatment, compared with the control group, the protein expression of cleaved-caspase- 3 and Bax was up-regulated in the TMZ group and the TMZ and LY294002 group but the expression was obviously less in the LY294002 group. The up-regulation of cleaved-caspase-3 and Bax expression was more significant in the TMZ and LY294002 group than in the TMZ group $(\mathrm{P}<0.05)$. In addition, $\mathrm{p}-\mathrm{Akt}$ and $\mathrm{Bcl}-2$, which are associated with TMZ resistance, were evidently down-regulated compared with the single therapy groups.

\section{Discussion}

High-grade malignant gliomas, including glioblastoma and anaplastic astrocytoma, are among the most rapidly growing and devastating types of cancer $(14,21)$. Standard treatment consists of surgery followed by radiotherapy and chemotherapy with alkylating agents $(2,3)$. Alkylating agents, including carmustine, lomustine, and TMZ, readily cross the blood-brain barrier and have shown the most activity against malignant glioma. However, despite the ability of these agents to achieve therapeutic concentrations in the brain, malignant gliomas are often resistant to alkylating agents (14). Many mechanisms of resistance to alkylating agents have been discovered, such as MGMT repair, mismatch repair pathway defection and anti-apoptotic protein activation (14).

Among these mechanisms, anti-apoptotic protein activation has received the most attention. Nagane et al (22) and Weller et al (23) showed that up-regulation of either Bcl-2 or EGFR expression in glioma cell lines was correlated with drug resistance and decreased apoptotic response in vitro. Caporali et al demonstrated that Akt was activated in an ataxia telangiectasia and $\mathrm{Rad} 3$ related-dependent manner in response to TMZ and conferred protection against druginduced cell growth inhibition (24). These results strongly support the hypothesis that clinical benefits are obtained by combining TMZ with inhibitors of the Akt pathway. Therefore 
a combinatorial approach of PI3K/Akt inhibition is urgent. In the present study, we focused on the synergistic mechanism of LY294002, a potent inhibitor of phosphoinositide 3-kinases, to enhance the cytotoxicity of TMZ. Our results revealed that LY294002 combined with TMZ more significantly inhibited tumor cell growth, invasion and induced apoptosis compared with TMZ used alone.

To date, LY294002 has been used in basic research to treat various types of cancer. There have been only a few reports of laboratory investigations on the effect of LY294002 in gliomas. Opel et al showed that PI3K inhibition by LY294002 sensitized primary cultured glioblastoma cells obtained from surgical specimens to TRAIL or chemotherapy-induced cell death (25). Han et al established that inactivation of PI3K/Akt signaling by LY294002 inhibited glioma cell growth through modulation of $\beta$-catenin-mediated transcription (26). We found that LY294002 produced a dose-dependent reduction in the proliferation of glioma cells. After exposure to TMZ $(10 \mu \mathrm{M})$ and LY294002 $(5 \mu \mathrm{M})$ for $48 \mathrm{~h}$, the number of Annexin V-positive tumor cells increased significantly, indicating an increase in apoptosis. The effect of LY294002 was similar to previous studies. Futhermore, we detected the expression of cleaved-caspase- 3 and Bax, both of which play crucial roles in the process of apoptosis, at the translational level. The results of immunblotting showed that the expression levels of cleaved-caspase-3 and Bax were up-regulated in both the TMZ alone group and the TMZ and LY294002 group, while the expression levels in the TMZ and LY294002 combination group were more significant. Hence we conclude that the down-regulation of p-Akt may facilitate the process of TMZ-mediated apoptosis.

To conclude, down-regulation of the PI3K/Akt pathway enhances the cytotoxicity of TMZ. The synergistic mechanism is mediated by down-regulation of p-Akt and Bcl-2, which leads to reinforced apoptosis upon combined treatment of TMZ and LY294002 in vitro.

\section{Acknowledgements}

This study was supported by the China National Natural Scientific Fund (nos. 30971136 and 81001128), the Program for New Century Excellent Talents in University (NCET07-0615), the Tianjin Science and Technology Committee (09JCZDJC17600).

\section{References}

1. Van Meir EG, Hadjipanayis CG, Norden AD, Shu HK, Wen PY and Olson JJ: Exciting new advances in neuro-oncology: the avenue to a cure for malignant glioma. CA Cancer J Clin 60 : 166-193, 2010.

2. Stupp R, Mason WP, van den Bent MJ, et al: Radiotherapy plus concomitant and adjuvant temozolomide for glioblastoma. N Engl J Med 352: 987-996, 2005.

3. Butowski NA, Sneed PK and Chang SM: Diagnosis and treatment of recurrent high-grade astrocytoma. J Clin Oncol 24: 1273-1280, 2006.

4. Kamiryo T, Tada K, Shiraishi S, Shinojima N, Kochi M and Ushio Y: Correlation between promoter hypermethylation of the O6-methylguanine-deoxyribonucleic acid methyltransferase gene and prognosis in patients with high-grade astrocytic tumors treated with surgery, radiotherapy, and 1-(4-amino-2-methyl5-pyrimidinyl)methyl-3-(2-chloroethyl)-3-nitrosourea-based chemotherapy. Neurosurgery 54: 349-357, 2004.
5. Verhaak RG, Hoadley KA, Purdom E, et al: Integrated genomic analysis identifies clinically relevant subtypes of glioblastoma characterized by abnormalities in PDGFRA, IDH1, EGFR, and NF1. Cancer Cell 17: 98-110, 2010.

6. Noushmehr H, Weisenberger DJ, Diefes K, et al: Identification of a $\mathrm{CpG}$ island methylator phenotype that defines a distinct subgroup of glioma. Cancer Cell 17: 510-522, 2010.

7. Bredel M, Scholtens DM, Harsh GR, et al: A network model of a cooperative genetic landscape in brain tumors. JAMA 302: 261-275, 2009.

8. Brennan C, Momota H, Hambardzumyan D, et al: Glioblastoma subclasses can be defined by activity among signal transduction pathways and associated genomic alterations. PLoS One 4: e7752, 2009.

9. Brennan C, Momota H, Hambardzumyan D, et al: $\gamma$-secretase inhibitors enhance temozolomide treatment of human gliomas by inhibiting neurosphere repopulation and xenograft recurrence. Cancer Res 70: 6870-6879, 2011.

10. Sunayama J, Sato A, Matsuda K, et al: Dual blocking of mTor and PI3K elicits a prodifferentiation effect on glioblastoma stem-like cells. Neuro Oncol 12: 1205-1219, 2011.

11. Cloughesy TF and Mischel PS: New strategies in the molecular targeting of glioblastoma: how do you hit a moving target? Clin Cancer Res 17: 6-11, 2010.

12. Chamberlain MC: Temozolomide: therapeutic limitations in the treatment of adult high-grade gliomas. Expert Rev Neurother 10: $1537-1544,2010$

13. Roos WP, Batista LF, Naumann SC, et al: Apoptosis in malignant glioma cells triggered by the temozolomide-induced DNA lesion O6-methylguanine. Oncogene 26: 186-197, 2007.

14. Sarkaria JN, Kitange GJ, James CD, et al: Mechanisms of chemoresistance to alkylating agents in malignant glioma. Clin Cancer Res 14: 2900-2908, 2008.

15. Dinnes J, Cave C, Huang S and Milne R: A rapid and systematic review of the effectiveness of temozolomide for the treatment of recurrent malignant glioma. Br J Cancer 86: 501-505, 2002.

16. Friedman HS, Kerby $\mathrm{T}$ and Calvert H: Temozolomide and treatment of malignant glioma. Clin Cancer Res 6: 2585-2597, 2000.

17. Chang SM, Theodosopoulos P, Lamborn K, et al: Temozolomide in the treatment of recurrent malignant glioma. Cancer 100: 605-611, 2004.

18. Kubiatowski T, Jang T, Lachyankar MB, et al: Association of increased phosphatidylinositol 3-kinase signaling with increased invasiveness and gelatinase activity in malignant gliomas. J Neurosurg 95: 480-488, 2001.

19. Shin JY, Kim JO, Lee SK, Chae HS and Kang JH: LY294002 may overcome 5-FU resistance via down-regulation of activated p-Akt in Epstein-Barr virus-positive gastric cancer cells. BMC Cancer 10: 425, 2010.

20. Ikezoe T, Nishioka C, Bandobashi K, et al: Longitudinal inhibition of PI3K/Akt/mTOR signaling by LY294002 and rapamycin induces growth arrest of adult T-cell leukemia cells. Leuk Res 31: 673-682, 2007.

21. Stupp R, Gander M, Leyvraz S, Newlands E: Current and future developments in the use of temozolomide for the treatment of brain tumours. Lancet Oncol 2: 552-560, 2001.

22. Nagane M, Coufal F, Lin H, Bogler O, Cavenee WK and Huang HJ: A common mutant epidermal growth factor receptor confers enhanced tumorigenicity on human glioblastoma cells by increasing proliferation and reducing apoptosis. Cancer Res 56: 5079-5086, 1996.

23. Weller M, Malipiero U, Aguzzi A, Reed JC and Fontana A: Protooncogene bcl-2 gene transfer abrogates Fas/APO-1 antibody-mediated apoptosis of human malignant glioma cells and confers resistance to chemotherapeutic drugs and therapeutic irradiation. J Clin Invest 95: 2633-2643, 1995.

24. Caporali S, Levati L, Starace G, et al: AKT is activated in an ataxia-telangiectasia and Rad3-related-dependent manner in response to temozolomide and confers protection against druginduced cell growth inhibition. Mol Pharmacol 74: 173-183, 2008.

25. Opel D, Westhoff MA, Bender A, Braun V, Debatin KM and Fulda S: Phosphatidylinositol 3-kinase inhibition broadly sensitizes glioblastoma cells to death receptor- and drug-induced apoptosis. Cancer Res 68: 6271-6280, 2008.

26. Han L, Yang Y, Yue X, et al: Inactivation of PI3K/AKT signaling inhibits glioma cell growth through modulation of $\beta$-cateninmediated transcription. Brain Res 1366: 9-17, 2010. 